PROSTATE CANCER

\title{
Inhibiting initiation targeting BMI1 is effective
}

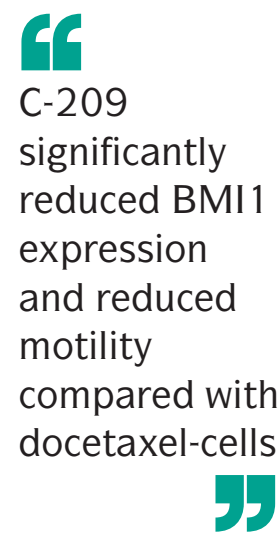

Inhibition of polycomb complex protein BMI-1 (BMI1) in prostate self-renewing tumour-initiating cells (TICs) with small-molecule post-transcriptional inhibitors reduces colony formation in vitro and tumour initiation in vivo, according to new data published in Clinical Cancer Research. Thus, targeting BMI1 in TICs could be an effective strategy for treating prostate cancer.

BMI1 regulates stem cell self renewal, has a key role in prostate cancer initiation and progression, and its downstream targets are associated with therapy resistance. Bansal and collegues show that expression of BMI 1 is increased in prostate cancer cell lines and that downregulation of BMI1 in vitro is associated with decreased cell motility, clonogenic capacity, and survival, providing a rationale for targeting this protein.

A high-throughput screen of $>200,000$ small molecules identified seven that target the post-translational control mechanisms of BMI1, three of which (C-209, C-210, and C-211) had a significant effect on TICs. In vitro analysis showed that these molecules are highly specific in their targeting of BMI1.

Treatment of human prostate cancer cell lines with C-209 induced cell cycle accumulation, reduced the number of cells in $\mathrm{S}$ phase, and induced a dose-dependant reduction in BMI1 expression. C-209 and C-211 diminished the number of secondary or tertiary spheroids that formed from single cells that has been collected from primary spheroids, indicating that these molecules can reduce the self-renewing capacity of these cells. Treatment of the normal prostate epithelium cell line RWPE1 and human haematopoietic stem and progenitor cells with C-209 had minimal effects on these cells, indicating the effects of this molecule are cancer-cell specific. However, in vivo toxicology assays showed that high doses of C-210 or C-211 had adverse effects of zebrafish embryos, therefore, only C-209 was taken forward for further analysis.

Treatment of primary patient-derived prostate cancer cells with C-209 significantly reduced BMI1 expression and reduced motility compared with docetaxel-treated cells. Patient-derived cells treated with C-209 and then replated formed significantly fewer colonies than cells treated with docetaxel.

In vivo, patient-derived xenografts grafted in to zebrafish and treated with C-209 showed tumour shrinkage, and pretreatment of xenografts with C-209 resulted in fewer tumours forming than for xenografts that had been pretreated with docetaxel.

These results suggest that molecules targeting BMI1 could specifically target prostate TICs and could be an effective prostate cancer therapy.

Louise Stone 